\title{
Yin Li: "a decade of perseverance" in promoting Enhanced Recovery after surgery in esophageal cancer patients
}

Submitted Apr 18, 2018. Accepted for publication Apr 25, 2018.

doi: $10.21037 /$ jtd.2018.05.05

View this article at: http://dx.doi.org/10.21037/jtd.2018.05.05

\section{Prologue}

We first met Prof. Yin $\mathrm{Li}$ in his office. He was wearing a doctor's white coat, a tie, and a badge, with a pen in the pocket of his coat, appearing professional and friendly. The office was crowded but clean and tidy, showing the occupant's good taste. The desk was covered with various items, including two large stacks of documents in addition to the computer. The bookcase behind the desk was full of medical books, and on the wall across the desk and above the sofa in the reception area was an ink painting of lotuses (Figure 1). In the corner of the room, vines of Jade Pothos were growing vigorously, framing the borders of the ink painting in a most pleasant way.

"I love lotuses, and I also like paintings of them." Prof. Li's father is an art teacher. "When I was young, my father wanted me to learn to paint, but I didn't like it, so I was buffeted." Although he did not become a painter, he likes paintings under his father's influence.

Kind, talkative, and humorous were our first impressions of Prof. Li. He was not pretentious and spoke matter-offactly; it was like we were listening to a family member telling a story. From daytime to evening, the interview lasted more than two hours, and when it ended, lights had already lit up the city. During the interview, we mostly listened and were inspired. As we were leaving, several family members of a patient began knocking on his office door, and Prof. Li continued the conversation with them.

\section{Introduction}

When he was 30 years old, Yin Li was daring but meticulous and curious; at the age of 40, he became the "helmsman" of the Department of Thoracic Surgery of Henan Provincial Cancer Hospital, being more open and tolerant and encouraging innovation. Now he is 50 years old, which, according to the sage, is the time a person perceives his destiny and wishes to remove the "excessive reputation", to perform "subtraction", and to concentrate on the things he

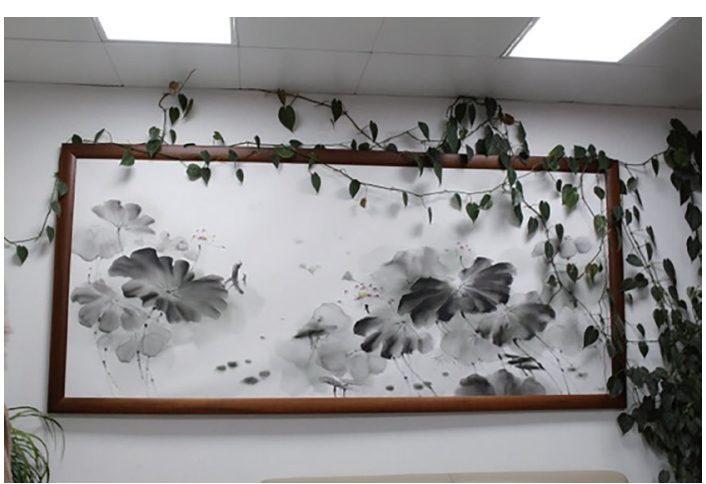

Figure 1 The ink painting in Prof. Li's office.

likes to do.

In 2007, Yin Li, at the age of 40 years old, was elected as the youngest director of the Department of Thoracic Surgery of Henan Provincial Cancer Hospital. In the same year, the concept of Enhanced Recovery after surgery (ERAS) was introduced in China and applied to general and gastrointestinal surgeries. Yin $\mathrm{Li}$ was the first person in China who advocated the idea of ERAS for esophageal cancer, becoming one of the earliest experts advocating for Enhanced Recovery after thoracic surgery. He devoted himself to the study for 10 years to promote ERAS for esophageal cancer patients, and for the first time in the world, he accomplished "non-tube no fasting" therapy for ERAS in patients with esophageal cancer.

\section{Expert's introduction}

Prof. Yin Li (Figure 2) is the vice president of Henan Provincial Cancer Hospital, director of the Department of Thoracic Surgery, advisor of doctoral students, and a specialist in surgical treatment of esophageal cancer. Prof. $\mathrm{Li}$ is an expert who has received a special allowance from the State Council, an academic and technological leader in Henan Province, and a member of the Royal College of Surgeons of the United Kingdom. He specializes 


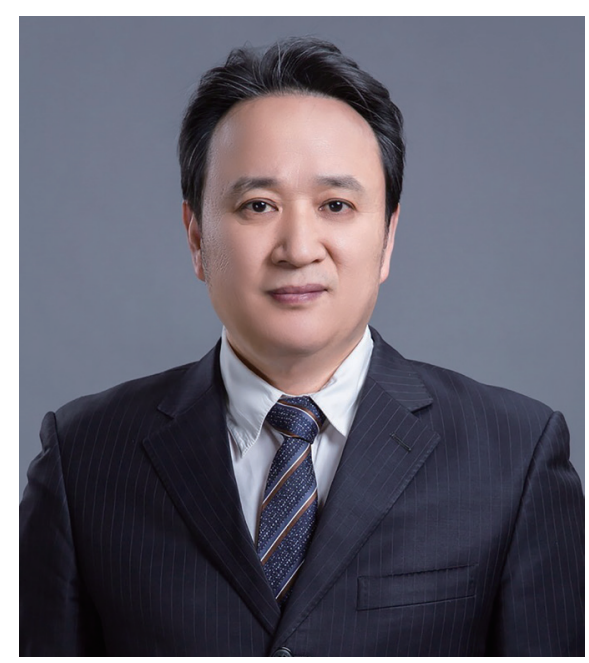

Figure 2 Prof. Yin Li.

in routine diagnosis and surgical treatment, minimally invasive treatment, and multidisciplinary comprehensive treatment of benign and malignant thoracic tumors, such as esophageal cancer, lung cancer, cardiac cancer, and thymic tumors. He has pioneered the clinical study of esophageal cancer ERAS models and related technologies worldwide, developed the "non-tube no fasting" ERAS model for esophageal surgeries, and was the first person proposing and achieving a new surgical treatment that enables patients to avoid suffering from the use of nasogastric feeding tube after surgery, obtain food by oral feeding, walk one day after surgery, and be discharged 5-7 days after surgery.

\section{As a rookie doctor}

Yin Li was born in Nanzhao County, Henan Province, where medical condition was dire, that is why his father encouraged him to become a medical doctor. Reluctant at first, he eventually entered Henan Medical University to become a medical student. After graduating from the medical school in 1989, Yin Li started his work in Henan Provincial Cancer Hospital. At the beginning he wanted to become a general surgeon, but eventually he became a thoracic surgeon. During his internship, because of his CCP party membership, Li was assigned to collate the archives and, by chance, read the archives of Prof. Lingfang Shao, an internationally renowned thoracic surgeon, which consisted of three volumes detailing the personal history of Prof. Shao and reflecting a wealth of perseverance, his ups and downs, and his pursuit of perfection. Having read these archives, Li was deeply impressed and determined to follow in the footsteps of Prof. Shao and work in the field of thoracic surgery. Fortunately, Prof. Shao accepted Yin Li as one of his apprentices.

In Yin Li's first few years working in the Department of Thoracic Surgery of Henan Provincial Cancer Hospital, the spirit of innovation and international perspective were not yet buzzwords. In 2003, the International Conference of Esophageal Surgery was held in Zhengzhou, which attracted a large number of internationally renowned experts on esophageal surgery. At the banquet table after the meeting, an expert voiced his opinion on the status quo of the field of thoracic surgery in China and the lack of innovation, which had a great impact on Yin Li. "Retrospective analysis is less valuable than innovation. Only with innovation can we move forward; if we always imitate, we will always be left behind." He spoke with Prof. Shao about his ideas, and Prof. Shao agreed with him. However, the road leading to innovation is long and winding.

In 2004, Yin Li graduated from Huaxi Medical Center of Sichuan University and became the first MD in Henan Provincial Cancer Hospital. Young and aspiring, he was keen on attending academic conferences to broaden his horizons. At that time, the department could not afford sending doctors to attend conferences for academic exchanges, but Yin Li was so determined that he attended at his own expense because he believed that only by learning continuously and trying persistently would he be able to develop a better method. Yin Li acquired skills from his peers on all occasions, e.g., conferences, exchange activities, and surgical demonstrations, among others. Back in the hospital, he started trying out new methods that were not written in textbooks. For example, when left thoracotomy was the mainstream procedure, Yin Li went to Fujian and learned how to perform right thoracotomy from Prof. Zhilian She at Fujian Provincial Tumor Hospital and continued his studies to improve the newly learned procedure.

In 2007, Fudan University Cancer Hospital invited Prof. James D. Luketich, an internationally celebrated expert from the US on minimally invasive esophageal surgeries, to demonstrate laparoscopic surgery. It was the first time that Yin Li watched minimally invasive laparoscopic surgery, and he was immensely impressed by this advanced surgical method and concluded that it must be the trend of the future. Back in the hospital, he immediately submitted an application to the hospital to purchase endoscopic instruments and introduce endoscopic surgery, but the application did not receive an approval after an entire year. Thus, he paid from his own money, a total of 180,000 CNY, 


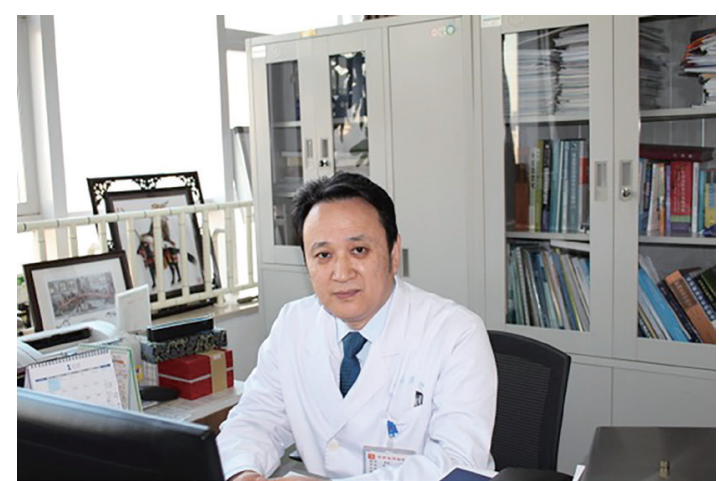

Figure 3 Prof. Li in his office.

to purchase endoscopic instruments and equipment, went to computer city to buy a monitor, and purchased chickens and rabbits from a farmer's market so that he could practice endoscopic surgeries at his home. When asked why he was so determined to learn laparoscopic surgery at that time, Li said, "We had no alternative; Henan Province has been devastated by esophageal cancer, and we must adopt advanced technologies to address this dire situation."

Henan Province has a high incidence of esophageal cancer, which is also known as a "poor man's disease" since it is highly prevalent in poverty-stricken areas. The treatment of esophageal cancer requires complex and delicate surgical procedures, and these patients experience a large amount of suffering due to the postoperative intubation and fasting. In 2007, the concept of ERAS was introduced to China, and $\mathrm{Li}$ was the first surgeon in the country to promote the concept of ERAS for esophageal cancer. He became the expert and pioneer of the country as he accepted the concept of ERAS and put it in clinical practice. It was also during this year that he became the chief of the Department of Thoracic Surgery of Henan Provincial Cancer Hospital. Perfection is not only achieved through hard work but can also be achieved by innovation. Yin Li (Figure 3), who is keen on investigating and innovating, devoted all his energy in the subsequent decade to the establishment of ERAS for esophageal cancer and a dedicated department.

\section{"It takes 10 years to grind a perfect sword."-exploration and innovation}

\section{Enhanced recovery—“Those who hold steadfast win"}

According to statistics, the morbidity and mortality of esophageal cancer in China account for more than half of those worldwide. Although China has a large team of esophageal surgeons, its academic innovations still lag far behind other areas of the world. Currently, the most common esophageal surgical procedures have been developed in Western countries. Faced with this situation, Yin Li believes that Chinese thoracic surgeons should make greater contributions to the treatment of esophageal cancer given that they are endowed with such a large number of cases and data sets.

The traditional surgical treatment of esophageal cancer requires the placement of a variety of tubes, such as chest tube, gastric tube, nasogastric tube, and jejunum stoma tube, as well as postoperative gastrointestinal decompression and fasting for several days, which not only causes suffering to the patient but also delays recovery. In 2007, the concept of ERAS proposed by the Danish surgeon Henrik Kehlet was introduced in China, which promotes the use of multimodality strategies to accelerate the patient's recovery; it was first applied in general surgery. The core concept of the idea is to use a series of evidence-based and optimized pre-, intra-, and post-operative measures to reduce physical and psychological trauma in the patient after surgery and thus effectively reduce the incidence of complications, shorten the hospital stay, lower the hospital readmission rate and risk of death, improve the prognosis, reduce medical costs, and ultimately achieve the goal of Enhanced Recovery of the patient. After being introduced to the idea of ERAS, Yin Li proposed its application in esophageal cancer patients and led the team in its implementation.

It takes courage to be "the first person to eat a crab", and innovation often takes hundreds of times more effort than "introduction". To change the status quo of intubation and fasting in esophageal cancer treatment, over the past decade, Yin Li has been committed to the study of the ERAS model of esophageal cancer. He and his team have been continuously exploring and improving surgical techniques while constantly summarizing and improving the treatment routes that enable patients to accelerate their recovery. Finally, he succeeded in being the first in the world to achieve the "non-tube no fasting" ERAS model for esophageal surgeries, a new surgical treatment model that does not require gastric tube and feeding tube intubation. After surgery, patients can eat orally, walk one day after surgery for esophageal cancer and achieve standard clinical discharge 5-7 days after surgery.

The road to innovation is a long process of trying and improving. To achieve the goal of "no intubation" and "eating orally after a short time", i.e., ERAS with "nontube no fasting", the key technical problem to be solved is 


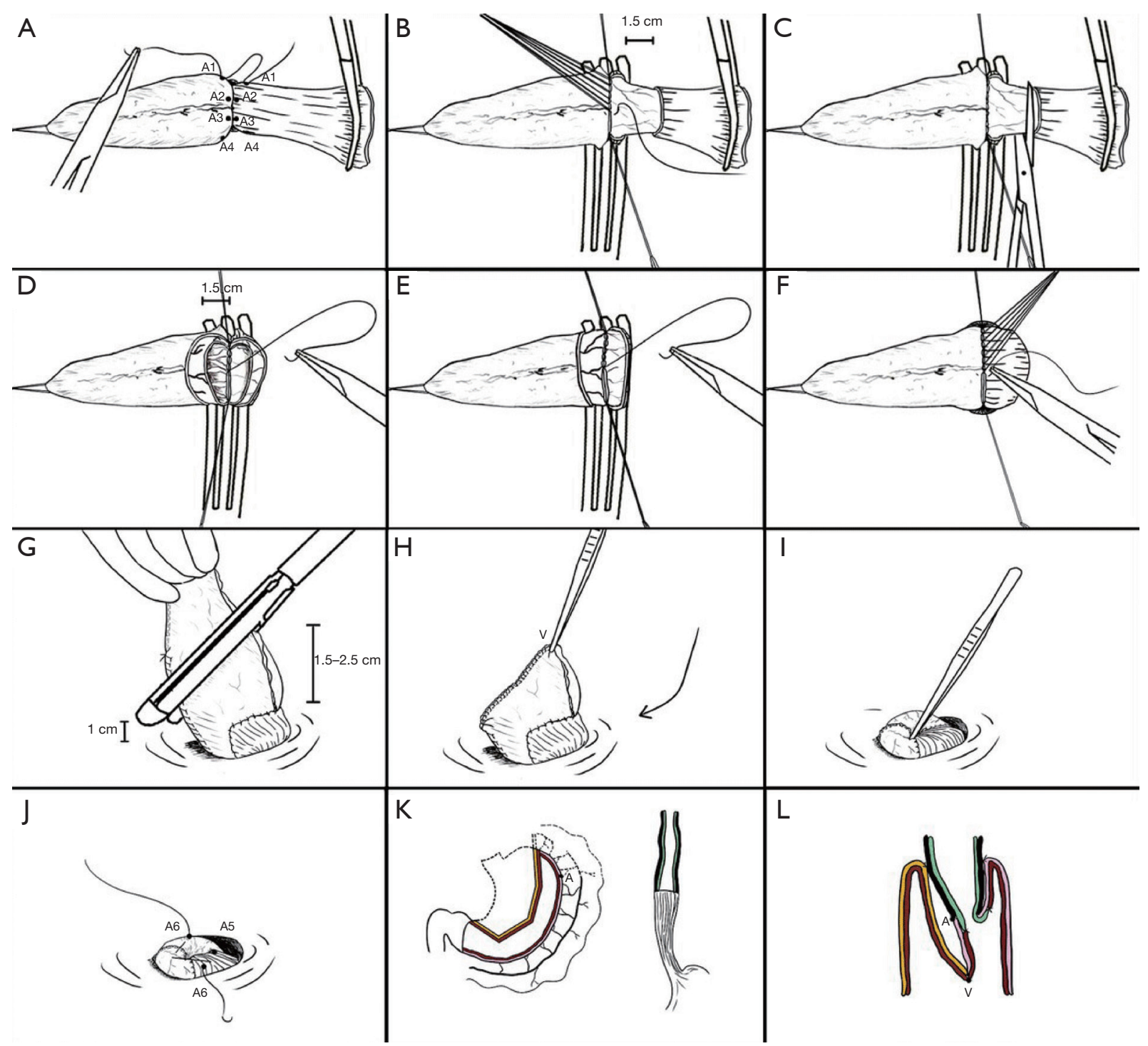

Figure 4 Diagrams of existing anastomotic procedures throughout the world (adopted from Li’s paper: http://jtd.amegroups.com/article/ view/4700/html).

to avoid anastomotic leakage. How can anastomotic leakage be avoided? Yin Li concluded, "On the one hand, it is about tension; the anastomotic stoma should be free of tension. On the other hand, it is about blood supply; we must ensure the stoma has a good blood supply." These are the two prerequisites that necessitate anastomotic procedures.

Li utilized the strategy of "finding a way by standing on the shoulders of giants", in which he listed all the existing anastomosis procedures in the world (1) (Figure 4). He then systematically analyzed these procedures and, through categorization and searching for patterns, concluded that although the procedures are diverse, they can be categorized into two types of anastomosis: single-layer anastomosis and double-layer anastomosis. After categorization, Li summed up their advantages and disadvantages and constantly improved them. "We have taken a lot of detours, tried all the methods, and even went abroad to watch other doctors' procedures." Ultimately, hard work paid off, and Yin $\mathrm{Li}$ invented "Li's anastomosis", an anastomotic procedure that allows him to achieve the goal of "non-tube no fasting" after esophageal surgery.

The concept of ERAS is covering the entire 
perioperative period and involves all aspects of pre-, intra-, and post-operative procedures. Yin Li's team has developed a set of effective ERAS concepts for esophageal cancer and participated in the development of the first expert consensus on ERAS for esophageal cancer in China in 2016, which detailed the surgical treatment procedures for esophageal cancer in terms of pre-, intra-, and postoperative aspects. "Preoperatively, we should pay attention to the nutritional assessment and evaluations of respiratory, cardiovascular, and pulmonary functions. Surgical procedures are closely interconnected, and we must pay special attention to the surgical procedures to protect the functions of the tissues. Postoperatively, patient management, including medication, nursing, nutrition, and others, must be meticulous. It is impossible for the entire process to succeed without multi-disciplinary collaborations," Yin Li briefly summarized for us the content of the expert consensus. In addition, airway management is an important part of thoracic surgery, and even a small mishap can lead to an array of complications. Thus, in 2016, Yin Li was also actively involved in the development of "The multidisciplinary expert consensus on perioperative airway management".

Under the ERAS mode, the design and management of wards need to be conducted scientifically. The Department of Thoracic Surgery of Henan Provincial Cancer Hospital is one of the earliest pain management ERAS wards. In the department managed by Yin Li, patients are all in ERAS wards, and on each patient's bedside card, a pain score that is scored according to international pain criteria ( $0-10$ points) is indicated. Under a nurse's instruction, the patient performs self-assessments of pain, and if the score is greater than 3 points, a doctor is notified to manage the patient. On the wall of the ward, a colored ribbon that is dotted with butterflies and flowers is painted, and the distance between two butterflies is one meter, which can be used to measure the distance a patient walks each day. Patients are under nurses' instructions. For example, when eating, the patients are asked to chew the food 50 times before swallowing, the amount of water they drink per day is measured, and in case of coughing, they are carefully guided. Currently, the ward management has been standardized. "For ERAS, clinical treatment procedures require constant improvement." (Figure 5).

To achieve ERAS for esophageal cancer, patient trust and cooperation are very important, and preoperative education should be effectively executed. Regarding how to gain patient trust, Yin Li has his unique viewpoints, " $A$ doctor must generate awe in the patient and win the patient's respect from the bottom of the patient's heart, and then the doctor may succeed. In front of the patient, a doctor must be authoritative and charismatic to acquire the necessary authority over the patient, while being consistent." How can a doctor have authority over the patient? Yin Li believes that the most important thing is to have outstanding skill, consider issues from the patient's perspective, try his/her best, and seek the best treatment option for the patient. Whenever a doctor is $100 \%$ dedicated to a patient, they will naturally win the patient's respect and trust.

Identifying the best way of management of esophageal cancer is a process of pursuing perfection, in which the details determine success or failure. Yin Li noted that, " $A t$ present, patient care in China ends when the patient is discharged, which is far from sufficient. When the patient goes bome, the patient is still not completely recovered and needs guidance from nursing professionals during the entire recovery process. For example, there were cases in which an improper coughing method has caused the anastomotic stoma to tear open. Thus, the concept of ERAS cannot be confined to the perioperative period but should be extended to community healthcare system and family medicine so that the professional guidance is 'at the patient's fingertips', and ERAS can be realized." Moreover, Yin Li believes that for the treatment of esophageal cancer, surgical techniques and neoadjuvant therapy are the two future research directions. Intraoperative protection of the functions of certain organs such as the vagus nerve is very important for patient recovery, which is also Yin Li's future research direction.

\section{Department management_- “Altruists win”}

Yin Li clearly remembers November 30, 2007, when he assumed the directorship of the Department of Thoracic Surgery of Henan Provincial Cancer Hospital. During the bidding process, Prof. Shao, who had already retired, recommended Yin Li to the hospital. Prof. Shao is one of the founders of the department, devoted his entire life to the department, and firmly believed that Yin Li, full of the spirit of innovation and persistence, was capable of leading the department forward. After becoming the director, Yin Li spent a week laying out a five-year departmental development plan, in which ERAS was the core and was surrounded by a departmental discipline system constructed to enable the department "to be able to treat all kinds of diseases and not drive patients away". The detailed and reasonable planning was effective. In 2012, the Department of Thoracic Surgery of Henan Provincial Cancer Hospital was selected as a national key specialist department and 


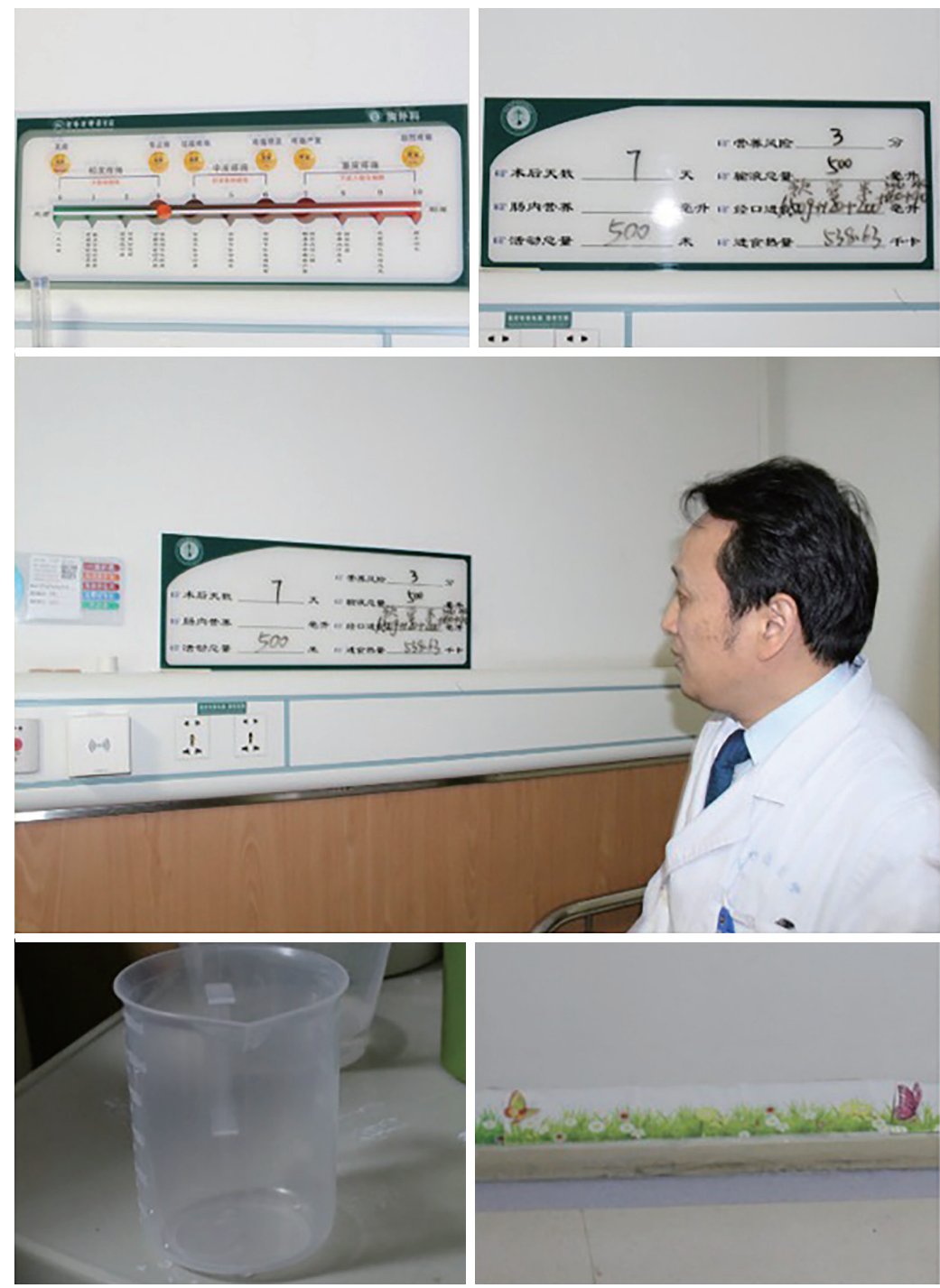

Figure 5 Prof. Li guides us through an ERAS pain management ward for patients recovering from thoracic surgery.

officially entered the country's leading echelon (Figure 6).

In this regard, Yin Li is particularly grateful for the trust and blessings of Prof. Shao and is determined to run the Department of Thoracic Surgery of Henan Provincial Cancer Hospital as a lifetime career, just as Prof. Shao has done during his tenure. Yin Li is passionate about the department, "To walk fast, walk alone; to walk far, walk with a team. Only with friendship, passion, and discipline can a department be successful." Their department is very disciplined, and the members perform their own duties in an orderly manner, making the department an organic entity. On Tuesdays, regardless of how busy the department is, no surgeries are arranged, and all members of the department congregate to discuss treatment options for each of their patients. Each doctor is asked to create a PowerPoint presentation on his/her patient's condition and propose his/ her own treatment plan, which is further discussed by all the members, so that treatment measures are optimized.

It is difficult to carry out the multidisciplinary team (MDT) consultation at the hospital level, so Yin Li took the lead and put it into practice in the Department of Thoracic Surgery. The department procured the video equipment, and at $7 \mathrm{am}$, seventy to eighty members would gather around and share a department-provided breakfast, then the consultation began. Doctors of internal medicine, radiation oncology, interventional radiology, imaging, and 


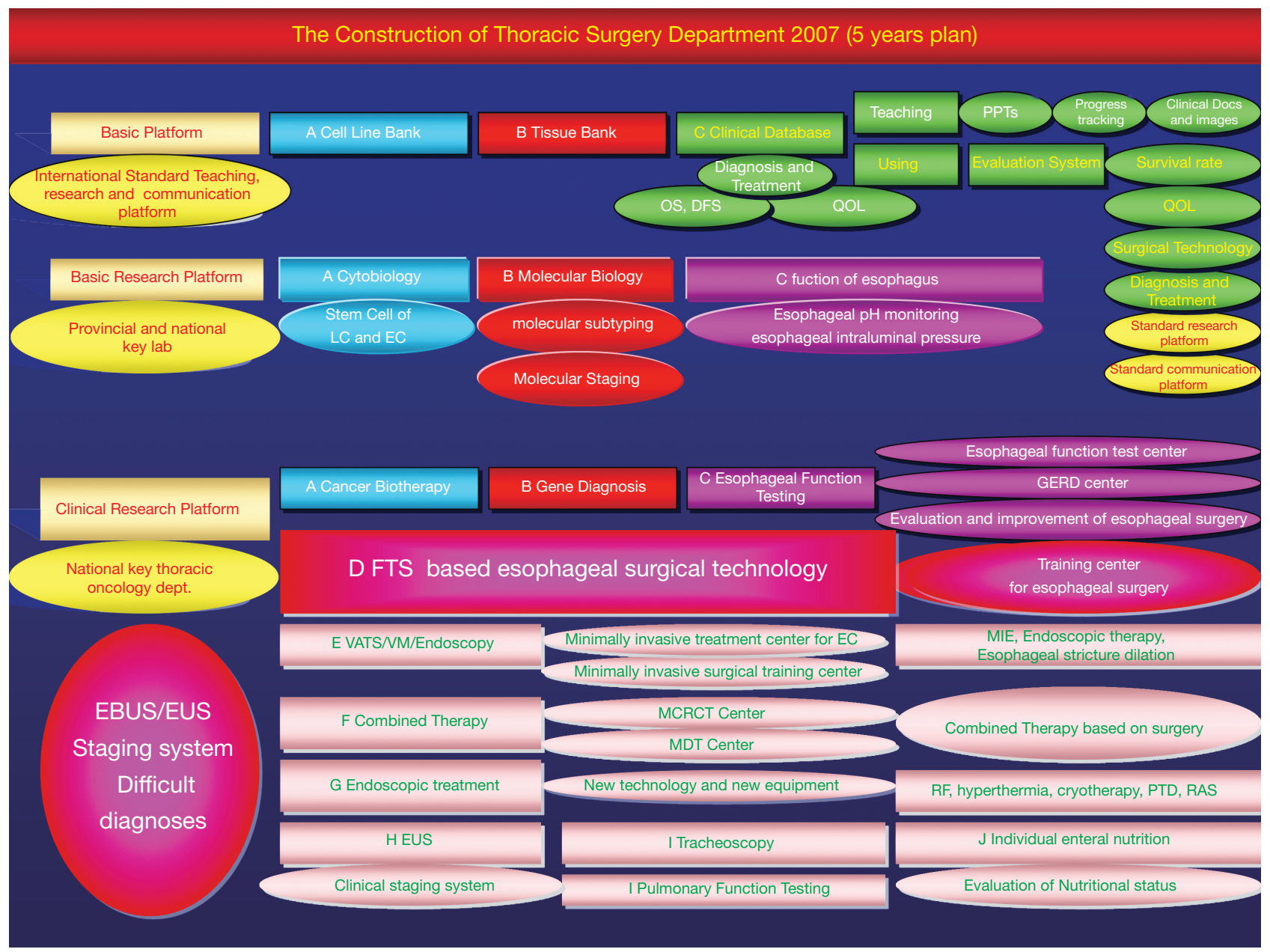

Figure 6 The developmental plan for the department that Prof. Li developed in 2007 ("X" indicates that it was not realized in 2012 but has since been accomplished).

pathology, among others, would discuss difficult cases and learn from each other. Yin Li believes that, during the MDT consultation, young doctors benefit the most by learning through listening and watching to accumulate the necessary knowledge.

"N minus one equals zero" is the expression that $\mathrm{Yin} \mathrm{Li}$ often uses to warn young doctors, since slight negligence during the treatment process can be lethal to the patient. "Surgeries must be performed wholeheartedly and are similar to the experience of trying to be a good person; if you give 100\%, you are bound to be handsomely rewarded." When performing surgeries, Yin Li is very careful and detail-oriented, and he pays special attention to protecting the tissues, since once a tissue is inadvertently damaged, the patient's recovery will be affected. "Surgeons like to use forceps and not pliers. When you push down on a pair of pliers, while it might look manly, cells are killed." When removing the stomach, Yin Li removes the stomach with both hands and places it on a wet gauze pad. Because the operating room is often rather cool, the stomach could acquire ischemia if it stays on the table top for too long, and Yin Li thus asks the nurse to keep pouring warm water on it to help maintain a normal temperature. The surgical procedures are interlocked, and surgeons must constantly strive for perfection so that patients are safe in their hands.

Regarding the cultivation of young doctors, Yin $\mathrm{Li}$ thinks that China is in an urgent need nowadays for a training system that fits its national conditions. China's traditional apprenticeship system has been dwindling, but it has not yet established new mature training models 
similar to those in Western countries, and a suitable environment that is conducive to the development of young doctors is still lacking. China is a major medical country, and the development of a training system with Chinese characteristics is urgently needed for the cultivation of outstanding doctors.

In 2017, 10 years had passed since Li's appointment. The disciplinary construction of the department had been gradually achieved, together with the attainment of competitive techniques, a basic research center with complete facilities, and training center that has received multiple accreditations. The department has developed from its original status of " 90 beds in two inpatient areas, less than 800 surgeries per year, 17 million CNY revenue per year, less than 20,000 CNY in research funding, and only a scalpel as departmental assets" to the current "national key clinical specialist department with 242 beds in 52 inpatient areas, 2,500 surgeries per year, 200 million CNY in revenue per year, nearly 10 million $C N Y$ in research funding, and nearly 30 million CNY for departmental assets." As a next step, Yin Li wants to improve the department's international recognition and create specialist departments with international impact, showcasing the skills of Chinese doctors.

"Those who are firm triumph, and those who are altruists win." This is a phrase Yin Li likes very much. "In the last decade, I have only published one paper, but with this paper, I am content."

\section{Eliminate "excessive reputation" and "do the subtraction"}

From 2007 to 2017, Yin Li devoted 10 years of effort to promoting ERAS for esophageal cancer and achieved breakthroughs at the international level; he has led the Department of Thoracic Surgery of Henan Provincial Cancer Hospital to constantly "compete to be at the top" in the country's leading echelon and toward the international arena.

In 2017, Yin Li reached the age of 50 and has earned many titles and honors, e.g., standing vice president of the hospital, special government allowance receiver, and Fellow of the British Royal College of Surgeons, among others, on which he does not place much value. "At the age of no confusion according to the sage's definition, I need to do more 'subtractions'." For him, too much "excessive reputation" is a distraction, making it impossible to focus on the things he wants to do.

Regarding his future plans, he said that at 50 years of age, he is experiencing a sense of urgency, and with only 10 years left at most as a professional, he has reached the age that he needs to do the "subtractions". In the past, he had to take all aspects into account and wanted to accomplish everything, but now he realizes that an individual's energy is limited. "For a surgeon, one is content with accomplishing one thing, and now, I only want to do the things that I enjoy doing."

\section{Interview}

JTD: When did you start learning the concept of ERAS after thoracic surgery?

Prof. Yin Li: In the $21^{\text {st }}$ century, three major new concepts in surgery were invented, which are ERAS, minimally invasive surgery, and injury control surgery. I heard about ERAS quite early at the $7^{\text {th }}$ National Scientific Conference of the Chinese Society for Thoracic and Cardiovascular Surgery in 2007, and Prof. Tianyou Wang, the former president of the Chinese Association of Thoracic Surgeons , introduced the concept of ERAS. At that time, ERAS was only a concept in thoracic surgery, but it had already been practiced in other surgical fields, such as the gastrointestinal and hepatobiliary fields. Later, at a conference on general surgery, I learned that the academician Jieshou Li of the General Hospital of the Nanjing Military Region had performed gastrointestinal surgery that allows the patient to eat orally a short time after surgery, and I began to pay attention. When I went back to the hospital, I searched for literature on ERAS and read everything I could find. The literature at the time addressed only some superficial items. I decided to start to attempt the method of ERAS and encourage patients to get their feeding orally at an earlier time post-surgery and increase their activities.

JTD: How did you initiate the pain management ward of ERAS after thoracic surgery in your department? Prof. Yin Li: We are one of the earliest departments in China that proposed the establishment of the pain management ward for thoracic surgery, and we have now established a set of management systems. The concept of ERAS has been integrated preoperatively, intraoperatively, and postoperatively, and it includes preoperative education and nutrition management, intraoperative incision and anesthesia drug selection, and postoperative medication and ward management.

In terms of ward management, we ask doctors and nurses to go to the ward and accompany the patient while walking in the ward to appreciate the patient's recovery-heart rate, 
gait, and so on-and to encourage the patient to eat while also teaching the patient how to eat and how to cough. There have been cases of anastomotic stoma ulcer due to a lack of education; for example, if a thin patient coughs hard, the force on the lung will exert great pressure on the stomach, especially when it contains gas. If the pressure is exerted on the site of the anastomotic stoma, then the stoma can be easily torn apart. Therefore, during ward management, we must pay attention to these details.

\section{JTD: What do you think is the best anastomosis method?}

Prof. Yin Li: There are many anastomosis approaches, and it is said that "the best method is the one that you are most familiar with"; i.e., doctors should use the most familiar method and then make improvements. Longitudinally speaking, this is reasonable that we should choose the method with which we are familiar and continuously improve our skills of the method, so the saying has scientific implications. However, crosswise speaking, the saying is incorrect. The best anastomosis method is the one with the lowest incidence of complications, and this is common sense.

JTD: In the "non-tube no fasting" model of ERAS after thoracic surgery, what are the follow-up studies that should be done?

Prof. Yin Li: In this regard, we would like to achieve breakthroughs first in overcoming difficulties and then systematically enriching the procedures. The so-called difficulties refer to life-threatening complications, such as anastomotic stoma ulcer and perforation. First, we must solve the problem of complications, and then we must work to protect functionality and provide nutritional support.

The protection of functionality refers to the emphasis on protecting cardiopulmonary and digestive functions, especially preservation of the vagus nerve and lung functions. If these functions are not properly protected, how to help patients achieve rapid recovery through postoperative management should be addressed, which is a topic necessitating more systematic and in-depth investigations.

Nutritional support is another big issue. The concept of ERAS spans the entire perioperative period, but it is clearly not sufficient to focus on only the perioperative period. For esophageal diseases, the patient's malnutrition can last 2 to 3 years or even longer; for example, when the vagus nerve is damaged, it will affect the patient throughout the remainder of their life. In Western countries, the recovery of patients after hospital discharge is adequately attended, but in China, insufficient attention is paid to it, and after the patient is discharged, the hospital no longer has any connection with the patient. We want to extend the concept of ERAS to the community and family, i.e., establish community healthcare system and family medicine to provide the patient with nutritional support and guidance. At present, we are still exploring how to help the patient recover after hospital discharge.

\section{JTD: What do you think about the direction of neoadjuvant treatment for esophageal cancer?}

Prof. Yin Li: Currently, there is no universally recognized neoadjuvant therapy mode in the world. First, the data in the East and the West cannot be unified; in Western countries, the data are abundant, but in these countries, the data are mostly about adenocarcinoma, while in Asia, squamous cell carcinoma is the most prevalent condition. Thus, we cannot blindly copy the data obtained in Western countries. Second, higher-level, randomized, controlled clinical studies (RCT studies) are still lacking.

Based on the existing literature regarding neoadjuvant chemotherapy and neoadjuvant chemoradiotherapy, researchers are still disputing which one is more promising. The ChemoRadiotherapy for Esophageal cancer followed by the Surgery Study (CROSS) group, which is one of the most influential research groups in Europe and the US, advocates neoadjuvant chemoradiotherapy. However, based on our experience, chemotherapy is more beneficial to patients, and they demonstrate good compliance to this regimen. Especially through the use of new drugs, the effectiveness of chemotherapy has gradually manifested, and we are currently conducting clinical studies on neoadjuvant chemotherapy. However, ultimately, the effectiveness of chemotherapy and chemoradiotherapy will likely require us to wait for another five years or so before we can draw relatively convincing conclusions.

JTD: You participated in the development of the "Chinese Expert Consensus Statement on Multidisciplinary Perioperative Airway Management (2016 Version)". Please tell us about the consensus development process. What important roles do you think this expert consensus plays in the clinical practice of thoracic surgeons?

Prof. Yin Li: We have conducted many discussions on developing this consensus, and the development of the consensus is a process from unawareness to awareness 
and from no systematic to systematic methodology. We have shared numerous cases at the meetings, including the ERAS ones. In the past, we did not pay sufficient attention to airway management, and with investigation, we have attached increasing importance to perioperative airway management because if it is not properly done, many lung complications arise. Whether it is lung cancer surgery or esophageal cancer surgery, the most common postoperative complication is pneumonia. When the lung is involved in thoracic surgery, airway management is undoubtedly very important. Moreover, it is also a process leading from a lack of systematic management to systematic management. In the past, we were only concerned with how to treat pneumonia when after it developed, but now we want to prevent the development of pneumonia. Preoperative assessment of the patient's heart and lung function helps to infer the risk of pneumonia, allowing early prevention arrangements. Intraoperatively, protection is strengthened. Postoperatively, physical and chemical intervention measures are promptly applied to high-risk patients who have not yet manifested symptoms. In discussions, the idea of ERAS was continuously integrated to systematize and streamline the measures. The consensus offers a systematic and clear pathway as well as adequate guidance on the clinical practices of surgeons.

\section{JTD: When you decided to create "specialist} department with an international impact", what are the criteria? What is your vision?

Prof. Yin Li: First, the most fundamental aspect is to have the world's leading technology; second, attending international conferences; third, the capability to hold unique international conferences; fourth, becoming an icon; fifth, which is the most difficult part, attracting patients from other countries to seek treatment at our department.

I hope we can present the technology of Chinese doctors to the outside world and attract foreign counterparts to visit and communicate and foreign patients to seek treatment. For example, heart surgeries in India are good and lowcost, giving rise to medical tourism. Thoracic surgeries in China are also first-rate and less expensive than those in developed countries. In this regard, China has many resource advantages; for example, regarding esophageal cancer, China has a large quantity of case and data resources, and with these huge patient resources, it will be very advantageous to develop training centers and conduct clinical studies.
If we can allow China's resource advantages full play, our country will benefit-peers will be motivated to visit and communicate, and our technologies will become internationalized. This will allow us to conduct international multi-center clinical studies so that we are not limited to multi-center clinical studies in China. More ambitiously, if we can build a sharing platform that includes training and databases, we can transform ourselves from a passive to an active player. If we can mine this resource, it will be beneficial for China as well as doctors in other countries since it can accelerate both training and research at home and abroad. Thus, I hope that we can establish our training system and share our databases with foreign countries, starting from attracting young doctors from foreign countries, and we can continuously broaden our international cooperation.

\section{JTD: Why did you propose to build a doctor training system with Chinese characteristics?}

Prof. Yin Li: At present, China's traditional mentoring model has basically vanished, but a new complete training system has not yet been established, so young doctors lack a suitable environment for career development, and the levels of trained doctors are inconsistent. In developed countries, the level of doctors tends to be homogenous; whether they are university professors, teaching doctors, or rural doctors, they are essentially at a similar level because they have completed a comprehensive training system, and medical students are evaluated at all levels before ultimately becoming a doctor.

China needs a huge team of physicians, but its training system is currently incomplete. Generally, when a young doctor is assigned to a department, he or she is mentored by a teacher, and how much the young doctor can learn depends on the teacher's teaching ability and the young doctor's learning ability. This kind of system is incomplete and insufficient. Therefore, China is urgently in need of a training system and a supervisory system that fit the national conditions. Medical students must pass different levels of rigorous training before they can become medical doctors. The US has the American College of Surgeons, the UK has the Royal College of Surgeons of England, and Japan has its own training system. Thus, China also needs to have its own College of Surgeons.

Video: Prof. Li's vision on the future development of ERAS after thoracic surgery and his message to AME and young doctors. Available online: https://v.qq.com/x/page/ u0537ggy4iv.html 


\section{Acknowledgements}

Thanks to Mmes. Siying Yan, Lili Liao and Mei Li from AME Publishing Company for their help in writing this article.

\section{Footnote}

Conflicts of Interest: The author has no conflicts of interest to declare.

Cite this article as: Zhao V. Yin Li: "a decade of perseverance" in promoting Enhanced Recovery after surgery in esophageal cancer patients. J Thorac Dis 2018;10(Suppl 11):S1297-S1307. doi: $10.21037 /$ jtd.2018.05.05

\section{References}

1. Zheng $Y$, Li Y, Wang $Z$, et al. A video demonstration of the Li's anastomosis- the key part of the "non-tube no fasting" enhanced recovery after surgery (ERAS) program for resectable esophageal carcinoma. J Thorac Dis 2015:7:1264-8.

(Science Editor: Vanessa Zhao, JTD, jtd@amepc.org) 